$\underline{\text { Review Articles }}$

\title{
Prognosis and Therapy When Acute Promyelocytic Leukemia and Other "Good Risk" Acute Myeloid Leukemias Occur as a Therapy-Related Myeloid Neoplasm
}

\author{
Richard A. Larson and Michelle M. Le Beau
}

Department of Medicine and Comprehensive Cancer Center, University of Chicago, Chicago IL, USA.

Correspondence to: Richard A. Larson, MD. Section of Hematology/Oncology, MC-2115, University of Chicago, 5841 S. Maryland Avenue, Chicago, IL 60637. Tel: 773-702-6783, FAX: 773-702-3002. E-mail: rlarson@medicine.bsd.uchicago.edu

Competing interests: The authors have declared that no competing interests exist.

Published: July 8, 2011

Received: May 24, 2011

Accepted: June 162011

Mediterr J Hematol Infect Dis 2011, 3: e2011032, DOI 10.4084/MJHID.2011.032

This article is available from: http://www.mjhid.org/article/view/8626

This is an Open Access article distributed under the terms of the Creative Commons Attribution License (http://creativecommons.org/licenses/by/2.0), which permits unrestricted use, distribution, and reproduction in any medium, provided the original work is properly cited.

Abstract: Treatment for a pre-existing condition using chemotherapy, radiation therapy, immunosuppressive therapy, or a combination of these modalities may lead to the devastating complication of therapy-related myelodysplastic syndrome or acute myeloid leukemia (t-MDS/tAML), collectively known as therapy-related myeloid neoplasm (t-MN). This disorder arises as a direct consequence of mutational events induced by the primary treatment. The outcomes for these patients have been historically poor compared to people who develop AML de novo. Currently comprising $10-20 \%$ of all cases of $A M L, t-M N$ is relatively resistant to conventional leukemia therapies, and is associated with short survival times. Median life expectancy from diagnosis is about 8-10 months in most series. Although the spectrum of cytogenetic abnormalities in t-AML is similar to AML de novo, the frequency of unfavorable cytogenetics, such as a complex karyotype or deletion or loss of chromosomes 5 and/or 7, is considerably higher in t-MN. Two distinct groups of patients with t-MN have been described. The more common subtype, seen in about $75 \%$ of patients, typically occurs 5-7 years after first exposure to alkylating agents or radiation, is often preceded by a myelodysplastic syndrome (MDS), and is frequently accompanied by clonal cytogenetic abnormalities such as the loss of all or part of chromosomes 5 or 7. Mutations of the P53 tumor suppressor gene are also common. The risk is related to total cumulative exposure over time to alkylating agents. In contrast, among individuals who develop t-AML after treatment with topoisomerase II inhibitors, the latency period to the development of t-AML is often only 1-3 years, antecedent MDS is rare, and gene rearrangements involving $M L L$ at $11 \mathrm{q} 23$ or $R U N X 1 / A M L 1$ at $21 q 22$ are common. It is now well recognized that APL and other subtypes of AML with balanced translocations sometimes occur as therapy-related myeloid neoplasms (t-MN) in patients who have previously received cytotoxic therapy or ionizing radiation therapy $(\mathrm{RT})$. The most of this review will focus on these "good risk" leukemias, i.e. those with APL or inv(16)/t(16;16) or t(8;21). 
Case Presentation: A 46-year-old woman was diagnosed with localized breast cancer. She underwent primary excision followed by adjuvant chemotherapy with doxorubicin and cyclophosphamide followed by paclitaxel; filgrastim was given to maintain a dose-dense schedule. Finally, she received radiation therapy to the left chest wall. No recurrence of breast cancer has been detected on routine follow up and imaging. However, 3 years later, she was noted to have pancytopenia. A bone marrow examination revealed a markedly hypercellular marrow with 90\% malignant promyelocytes and blasts. The karyotype was $46 X X$,t $(15 ; 17)$ in all 30 metaphase cells. Her cardiac left ventricular ejection fraction has declined to $40 \%$.

Is this therapy-related acute promyelocytic leukemia (t-APL)? Will it respond differently than de novo APL? Can it be treated successfully without exposure to additional anthracyclines or the use of hematopoietic stem cell transplantation (HCT)? Do "cytogenetically favorable" subsets of therapyrelated myeloid leukemia ( $\mathrm{t}-\mathrm{AML}$ ) have a different outcome than more typical cases of $\mathrm{t}$-AML with complex cytogenetic abnormalities?

The Syndrome of Therapy-Related Myeloid Neoplasm: Treatment for a pre-existing condition using chemotherapy, radiation therapy, immunosuppressive therapy, or a combination of these modalities may lead to the devastating complication of therapy-related myelodysplastic syndrome or acute myeloid leukemia (t-MDS/t-AML), collectively known as therapy-related myeloid neoplasm $(\mathrm{t}-\mathrm{MN}) .{ }^{1}$ This disorder arises as a direct consequence of mutational events induced by the primary treatment. The outcomes for these patients have been historically poor compared to people who develop AML de novo. Currently comprising $10-20 \%$ of all cases of AML, t-MN is relatively resistant to conventional leukemia therapies, and is associated with short survival times. ${ }^{1-3}$ Median life expectancy from diagnosis is about 8-10 months in most series. ${ }^{2}$ Although the spectrum of cytogenetic abnormalities in t-AML is similar to AML de novo, the frequency of unfavorable cytogenetics, such as a complex karyotype or deletion or loss of chromosome 5 and/or 7, is considerably higher in t-MN. ${ }^{2}$

Two distinct groups of patients with t-MN have been described. ${ }^{1,2,45}$ The more common subtype, seen in about $75 \%$ of patients, typically occurs 5-7 years after first exposure to alkylating agents or radiation, is often preceded by a myelodysplastic syndrome (MDS), and is frequently accompanied by clonal cytogenetic abnormalities such as the loss of all or part of chromosomes 5 or 7 . Mutations of the P53 tumor suppressor gene are also common. The risk is related to total cumulative exposure over time to alkylating agents. In contrast, among individuals who develop tAML after treatment with topoisomerase II inhibitors, the latency period to the development of t-AML is often only 1-3 years, antecedent MDS is rare, and gene rearrangements involving $M L L$ at $11 \mathrm{q} 23$ or RUNX1/AML1 at $21 \mathrm{q} 22$ are common. Risk is less clearly related to total cumulative dose but is associated with chemotherapy dose and schedule.
The Importance of Cytogenetic Abnormalities in Predicting Patient Outcomes: Within a series of 306 patients with t-MN studied at the University of Chicago, the median survival rate was approximately 7 to 9 months, and varied with karyotype. ${ }^{2}$ The longest median overall survival rates were seen in patients with normal karyotypes or recurring balanced rearrangements (approximately 11 months each). However, the incidence of unfavorable karyotypes was greater than $70 \%$. The shortest median survival was seen in patients with abnormalities of both chromosomes 5 and 7 (approximately 5 months). Only 24 patients $(8 \%)$ were alive 3 years after diagnosis. Patients with t-MN who responded to remission induction therapy but subsequently died from their primary malignancy were included in the survival analysis. The short survivals reported in this early series compared with more recent series described below reflects a higher percentage of patients who received only supportive care rather than undergo remission induction chemotherapy at that time.

Survival for patients receiving intensive remission induction chemotherapy varies according to cytogenetic risk group. Better outcomes are observed in t-MN patients with more favorable-risk karyotypes. A large comparative analysis reported by the German AML Cooperative Group included 93 patients with tAML and 1091 patients with de novo AML treated with standard AML induction therapy. ${ }^{6}$ Overall, the median survival was 10 months for patients with t-

AML compared to 15 months for patients with de novo AML $(\mathrm{P}=0.0007)$. Favorable, intermediate, and unfavorable karyotypes were observed in $26 \%, 28 \%$, and $46 \%$ of t-AML patients, and in $22 \%, 57 \%$, and $20 \%$ of de novo AML patients. The high frequency of adverse cytogenetics may explain to a large extent the unfavorable outcomes of patients with t-AML. Although favorable and unfavorable cytogenetics had 
Table 1. Survival according to cytogenetic risk group for patients with t-AML or de novo AML treated by the German AML Cooperative Group (AMLCG). ${ }^{7}$

\begin{tabular}{|l|c|c|c|c|c|}
\hline Karyotype & \multicolumn{2}{|c|}{ No. of patients (\%) } & \multicolumn{3}{c|}{ Median survival (months) } \\
\hline & $\mathrm{t}-\mathrm{AML}(\mathrm{n}=121)$ & de novo AML (n=1511) & $\mathrm{t}$-AML & de novo AML & $\mathrm{p}$ \\
\hline Favorable & $29(24)$ & $306(20)$ & 27 & 12 & 0.02 \\
\hline Intermediate & $34(28)$ & $903(60)$ & 6 & 7 & 0.19 \\
\hline Unfavorable & $58(48)$ & $302(20)$ & 12 & 0.006 \\
\hline
\end{tabular}

prognostic value in both patient groups, the survival of patients with t-AML was generally shorter than that of those with de novo AML within the same cytogenetic risk group. When updated to include 121 patients with t-AML, the median overall survival times for patients with t-AML with favorable, intermediate, and unfavorable cytogenetics were 27, 13, and 6 months, respectively (Table 1). ${ }^{7}$ For those with a favorable karyotype, the median survival was not yet reached after 5 years for the 306 de novo AML patients compared to 27 months for the 29 t-AML patients $(\mathrm{P}=0.02)$. Some of these t-AML patients appeared to be cured. Within the large intermediate risk cytogenetic group, no significant difference in survival was observed between the t-AML and de novo AML patients. An unfavorable karyotype predicated a very short survival in both groups of AML patients.

Armand et al analyzed the outcomes of 80 patients with therapy-related leukemia treated at the Dana Farber Cancer Institute in Boston. ${ }^{8}$ They found that cytogenetic abnormalities were the strongest predictors for overall survival. When adjusted for cytogenetic changes, the patients with t-AML did as well as patients with de novo AML, further emphasizing the importance of cytogenetic abnormalities in predicting severity of disease and outcomes.

Kayser et al recently reported on 200 patients with t-AML and compared their clinical and biological characteristics and outcomes with those of 2653 patients with de novo AML treated on 6 prospective multi-center trials by the German-Austrian AML Study Group. ${ }^{3}$ Seventy-five percent of the t-AML cases had an abnormal karyotype compared to $51 \%$ of the de novo cases, and $15 \%$ of the t-AML cases had either a $\mathrm{t}(15 ; 17)[2 \%], \mathrm{t}(8 ; 21)$ [5\%], or $\operatorname{inv}(16)$ or $\mathrm{t}(16 ; 16)$ [8\%]. The t-AML patients were older (median, 58 vs 53 years; $p<0.0001)$ and more often female $(68 \%$ vs $47 \% ; \mathrm{p}<0.0001)$. The median latency was 4.0 years (range, 0.3 to 44 years). The response to remission induction therapy was $63 \% \mathrm{CR}$ for t-AML and $67 \%$ for de novo AML $(\mathrm{p}=0.21)$. However, the outcome for $\mathrm{t}-$ AML patients was significantly inferior. Relapse-free survival at 4 years was $25 \%$ for t-AML and $40 \%$ for $d e$ novo AML $(\mathrm{p}<0.0001)$. In multivariable models adjusted for white blood cell counts, among patients with an inv(16) or $\mathrm{t}(16 ; 16)$, t-AML was a significant adverse prognostic factor for overall survival (hazard ratio $2.35 ; \mathrm{p}=0.04)$.

Factors that Influence Outcome in t-MN: Therapyrelated myeloid leukemia is generally a fatal disease. The life-threatening complications of this disorder are the result of persistent and profound cytopenias due to the failure of normal hematopoiesis regardless of the fraction of myeloblasts accumulating in the bone marrow or blood. There has been general agreement that patients with t-AML have shorter survivals than patients with de novo AML. Supportive care is still considered by many to be the standard management.

The survival of patients with t-MN is often poor despite prompt diagnosis and treatment. However, there is a paucity of prospective treatment data since these patients are most often excluded from frontline clinical trials. There are no randomized studies comparing standard AML therapy to other forms of treatment. A number of potential factors explain the poor outcome of patients with t-MN. Poor hematopoietic reserves can make the administration of standard AML therapy difficult. Because t-MN evolves in the milieu of chemotherapy, the malignant cells may be more drug-resistant than in de novo disease. Persistence of the primary malignant disease, particularly metastatic cancer or lymphoma, causes morbidity and mortality independent of the bone marrow failure caused by leukemia. Many patients have poor tolerance for the acute toxicity of treatment. Injury to organs and their vascular supply from prior treatment may compromise the ability of these patients to receive intensive chemotherapy or successful HCT. There may be depletion of normal hematopoietic stem cells as a consequence of previous therapy, so that these patients suffer prolonged cytopenias after induction chemotherapy. The bone marrow stroma may have been damaged, especially by therapeutic radiation to fields that include the pelvis or lumbosacral spine, so that it will not support regeneration of normal hematopoiesis. Patients with t-AML are often chronically immunosuppressed from prior disease or on-going therapy or may have dysfunctional and dysplastic phagocytes, and thus are often colonized with pathogenic or antibiotic-resistant bacteria and fungi. Following prior supportive care, patients may be 
refractory to additional transfusion support, and therefore, not good candidates for intensive myelosuppressive chemotherapy. Finally, the high frequency of unfavorable cytogenetic aberrations arising during or after chemoradiotherapy appears to result in the rapid emergence of chemotherapy resistance in t-AML stem cells. Relapses even after myeloablative chemoradiotherapy and allogeneic HCT are not uncommon.

Outcomes of t-MN Patients with "Good Risk" Cytogenetics: It is now well recognized that APL and other subtypes of AML with balanced translocations sometimes occur as therapy-related myeloid neoplasms $(\mathrm{t}-\mathrm{MN})$ in patients who have previously received cytotoxic therapy or ionizing radiation therapy (RT). ${ }^{1,39}$ The exact mechanism of the leukemogenic transformation remains to be determined. The remainder of this review will focus on "good risk" leukemias, i.e. those with APL or inv(16)/t $(16 ; 16)$ or $\mathrm{t}(8 ; 21)$.

Among 511 patients analyzed at the International Workshop on the Relationship of Prior Therapy to Balanced Chromosome Aberrations in TherapyRelated Myelodysplastic Syndromes and Acute Leukemia held in Chicago in 2000, chromosome 21q22 rearrangements were seen in $79(\sim 15 \%)$, inv(16) in 48 [9\%; 2 patients had $\mathrm{t}(16 ; 16)]$, and $\mathrm{t}(15 ; 17)$ in 41 $(8 \%) .{ }^{10}$ It was noted that $30 \%$ of $\mathrm{t}(15 ; 17)$ cases and $21 \%$ of inv(16) cases had received only RT as their primary treatment, and this was significantly more frequent than in the chromosome 11q23 (5\%) and $21 \mathrm{q} 22(7 \%)$ cases $(\mathrm{p}<0.001)$. The majority of patients in all subgroups had received alkylating agents. The chromosome 11q23 and 21q22 subgroups actually had the highest exposures to alkylating agents $(86 \%$ and $82 \%$, respectively); this was significantly greater than for the inv(16) and $t(15 ; 17)$ subgroups $(63 \%$ and $59 \%$, respectively). None of the t-APL patients had received the dioxypiperazine derivative, bimolane, once used in a Chinese study for treatment of psoriasis, and associated with $\mathrm{t}(15 ; 17)$.

The disease was overt t-AML in 38 of 48 patients (79\%) with an inv(16) and in 38 of 41 patients (93\%) with a $t(15 ; 17) .{ }^{11}$ Cases with inv(16) had a short latency period (median, 22 months) and a long median survival, with $45 \%$ surviving for 5 years. The median latency for $\mathrm{t}(15 ; 17)$ was 29 months, and the median survival 29 months. The primary disease for inv(16) cases was breast cancer $(\mathrm{n}=15)$, lymphoma(12), and other solid tumors $(16)$. For the $t(15 ; 17)$ cases, it was breast cancer (18),lymphoma (11),and other cancers (12). Twenty-six patients with inv(16) (54\%) and 17 with $t(15 ; 17) \quad(41 \%)$ had additional cytogenetic abnormalities; these were unrelated to age or survival in both subgroups. Trisomy 8 was the most common additional abnormality in both; $+21,+22$ and +13 were common in the inv(16) subgroup, and $-7 / \operatorname{del}(7 q)$ and $5 / \operatorname{del}(5 q)$ were present in a few cases in both subgroups.

Outcomes of Patients with t-MN and T(15;17) or Inv(16): Thirty-three of 39 patients (85\%) with an inv(16) obtained a complete remission (CR) after intensive chemotherapy, but 12 of these subsequently relapsed..$^{10}$ Four underwent HCT using bone marrow stem cells, and one underwent peripheral blood stem cell transplantation. All 5 of these patients remained in $\mathrm{CR}$ at the time of last follow-up. The 6 patients with inv(16) who did not respond to intensive chemotherapy were significantly older (median age, 62 years) as compared to the 33 responding patients (median, 44 years) $(\mathrm{P}=0.012)$.

Twenty-four of 35 patients $(68 \%)$ with a $t(15 ; 17)$ obtained a CR after intensive chemotherapy, and 6 patients subsequently relapsed. Two additional patients achieved a CR, but follow up data were missing. Two patients received an allogeneic HCT, and one was in $\mathrm{CR}$ at last follow-up. In the $\mathrm{t}(15 ; 17)$ subgroup, the 11 non-responding patients were similar in age to the 24 responding patients (medians, 48 and 50 years, respectively; $\mathrm{p}=0.99)$. Several patients with $\mathrm{t}(15 ; 17)$ were treated with all-trans-retinoic acid (ATRA, tretinoin), but information on the use of ATRA was recorded inconsistently in the Workshop database.

The median survival time was 29 months in both cytogenetic subgroups, and many patients became long-term survivors. There were no significant differences in survival among intensively treated patients with or without additional chromosome abnormalities (log-rank test, $\mathrm{p}=0.16$ for the $\operatorname{inv}(16)$ subgroup, and $p=0.30$ for the $t(15 ; 17)$ subgroup). The overall survival of $39 \operatorname{inv}(16)$ patients with follow up data who had previously received chemotherapy with or without RT $(n=33)$ for their primary disease was significantly better compared to patients who had received RT only $(\mathrm{n}=6)$ (log-rank test, $\mathrm{P}=0.03)$. The difference in survival between primary chemotherapy with or without RT and RT only was not statistically significant in the $\mathrm{t}(15 ; 17)$ subgroup $(\mathrm{P}=0.45)$.

In the inv(16) subgroup, patients less than 55 years old had improved survival when compared to older patients. The median survival for the 26 younger patients had not been reached, but was only 12 months for the 13 older patients ( $\log$-rank, $p=0.006$ ). A similar tendency, although not statistically significant, was observed in the $\mathrm{t}(15 ; 17)$ subgroup, with median 
survival times of 29 and 20 months in the younger $(\mathrm{n}=21)$ and older $(\mathrm{n}=15)$ cohorts $(\mathrm{p}=0.73)$, respectively.

Treatment of Patients with t-MN and t(15;17) or inv(16): In 2003, Beaumont and colleagues reported on 106 patients diagnosed with t-APL during the previous 20 years in 3 European countries; 80 were diagnosed within the previous 10 years. ${ }^{12}$ Primary disorders were predominantly breast cancer $(n=60)$, non-Hodgkin lymphoma (15), and other solid tumors (25). Thirty patients had received chemotherapy alone, 27 had received RT alone, and 49 had received both. Prior chemotherapy included at least one alkylating agent in 68 patients and at least one topoisomerase-II inhibitor in 61, including anthracyclines (30), mitoxantrone (28), and epipodophyllotoxins (19).Median latency was 25 months (range, 4 to 276 months). Characteristics of t-APL were generally similar to those of de novo APL. Survival was $59 \%$ at 8 years.

Several additional series of patients with t-APL have recently been published. Jantunen and colleagues in Finland reported on 5 cases; all had received prior RT; 4 had received topoisomerase-II inhibitors with multiagent chemotherapy. ${ }^{13}$ All achieved CR with ATRA plus chemotherapy. One died from metastatic breast cancer and one relapsed; 3 remain in continuous CR after a median of 28 months. Dayyani and coinvestigators at the MD Anderson Cancer Center reported on 29 patients with t-APL; 33\% had received only prior RT. ${ }^{14}$ Their median age was about 54 years compared with 42 years for a cohort of de novo APL patients treated at the same institution $(\mathrm{p}<0.001)$.

Otherwise, clinical features and outcomes were similar. The CR rate was $89 \%$ for the 19 with t-APL who received ATRA plus arsenic trioxide (ATO), and $70 \%$ for 10 who received ATRA plus chemotherapy $(\mathrm{p}=0.35)$. At last follow up, 15 patients $(52 \%)$ were alive; 9 had died from leukemia. Malhotra and colleagues in India treated 3 patients with t-APL with ATRA plus ATO for induction and consolidation. ${ }^{15}$ All 3 remain in molecular remission at 19-42 months.

In contrast to other subtypes of t-MN, patients who develop t-APL with $\mathrm{t}(15 ; 17)$ or those with inv(16) have treatment outcomes that are similar to AML that arises de novo with the same chromosomal rearrangements. The combination of ATRA plus arsenic trioxide is an effective treatment for t-APL and may be particularly useful for patients who have diminished cardiac function from prior anthracycline exposure or radiation. Although non-leukemia co-morbidities or persistent primary malignancy still impact on ultimate survival, their leukemia should be treated similar to de novo disease. ${ }^{16}$

Patients with t-MN and t(21q22): The Workshop identified $79 \mathrm{t}-\mathrm{MN}$ patients with balanced chromosome $21 \mathrm{q} 22$ translocations; $44(56 \%)$ had $\mathrm{t}(8 ; 21), 11$ as a sole abnormality and 33 in combination with other abnormalities. ${ }^{17,18}$ Median latency was 39 months overall. Patients had been treated for solid tumors (56\%), hematologic malignancy (43\%), and juvenile rheumatoid arthritis ( 1 case) with RT alone $(n=5)$, chemotherapy alone (36), or combined-modality therapy (38). All 5 who had received only RT had $\mathrm{t}(8 ; 21)$. Exposure to alkylating agents was significantly greater than for the inv(16) and $t(15 ; 17)$ subgroups $(\mathrm{p}<0.02$ for both). Exposure to topoisomerase-II inhibitors was significantly greater than in the $\mathrm{t}(15 ; 17)$ subgroup but similar to inv(16). Overt t-AML was present at diagnosis in $82 \%$ with $\mathrm{t}(8 ; 21)$. Median survivals were 17 months for the 11 with only $\mathrm{t}(8 ; 21)$ and 31 months for the 33 with $t(8 ; 21)$ plus other abnormalities $(\mathrm{p}=0.6)$. Mutations in $c$-KIT were not studied in these patients. ${ }^{19}$ Patients with $\mathrm{t}(8 ; 21)$ had a more favorable outcome than those with other $21 \mathrm{q} 22$ present at diagnosis in $82 \%$ with $\mathrm{t}(8 ; 21)$. Median survivals were 17 months for the 11 with only $\mathrm{t}(8 ; 21)$ and 31 months for the 33 with $t(8 ; 21)$ plus other abnormalities $(\mathrm{p}=0.6)$. Mutations in $c$-KIT were not studied in these patients. ${ }^{19}$ Patients with $\mathrm{t}(8 ; 21)$ had a more favorable outcome than those with other $21 \mathrm{q} 22$ rearrangements $(p=0.014)$. Overall, median survival for the $21 \mathrm{q} 22$ patients was 14 months, and for patients with $\mathrm{t}(8 ; 21)$ was 19 months.

Recommendations for Treatment of t-MN: Figure 1 shows a treatment algorithm for the management of patients who develop therapy-related myeloid neoplasms. Primary considerations are the patient's performance status, which reflects age, comorbidities, the status of the primary disease, and the presence of complications from primary therapy, as well as the clonal abnormalities detected in the t-MN cells. Mutations in $c$-KIT were not studied in these patients.

In general, t-MN patients should be encouraged to participate in prospective clinical trials that are appropriately designed for other AML patients with similar cytogenetic abnormalities. Patients who have an HLA-matched donor should be considered for allogeneic HCT, although patients with favorable karyotypes such as $t(15 ; 17)$ and inv(16) may do well with conventional intensive chemotherapy. 
Figure 1.

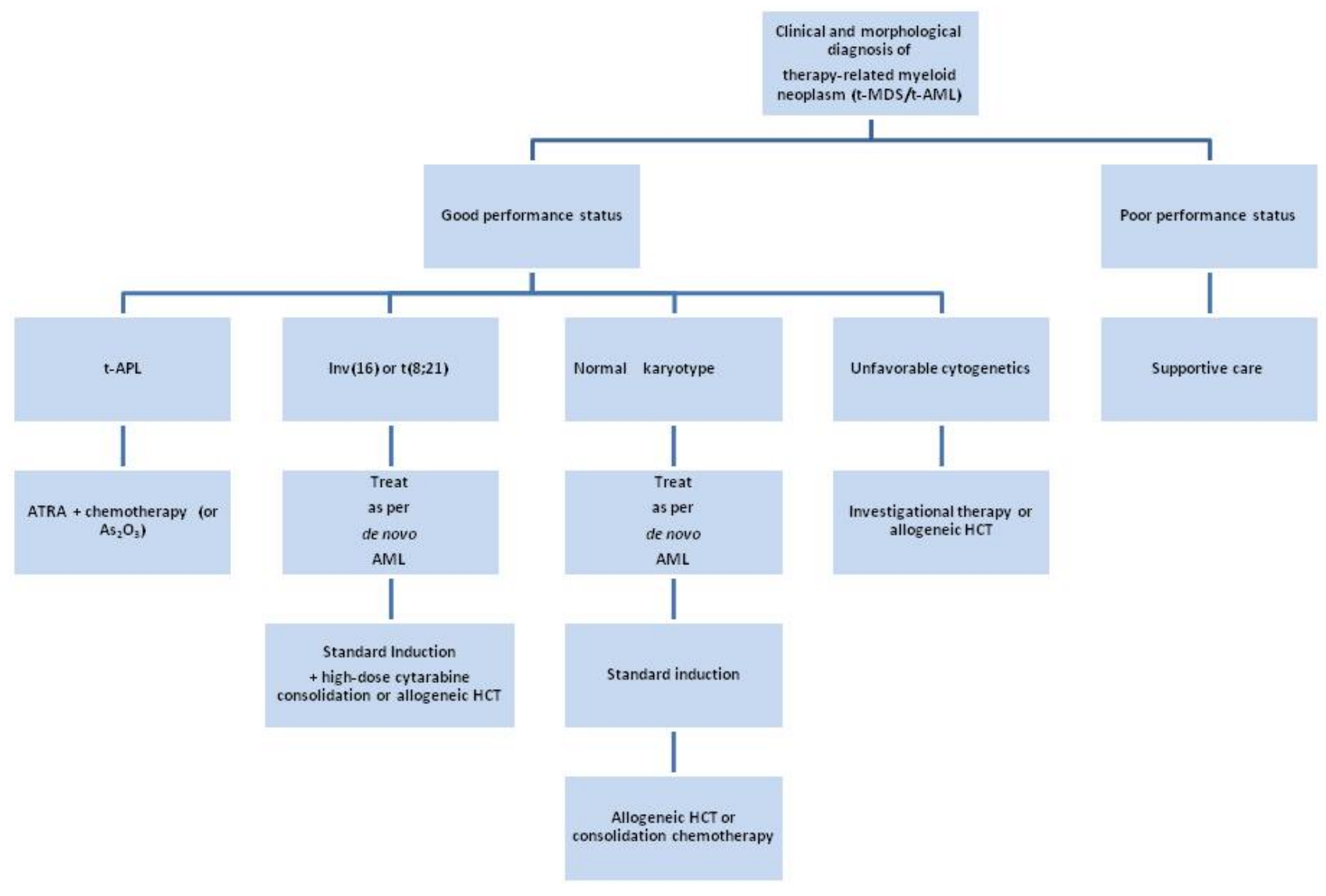

\section{References:}

1. Godley LA and Larson RA. Therapy-related myeloid leukemia. Semin Oncol 2008; 35(4):418-429. doi:10.1053/j.seminoncol.2008.04.012 PMid:18692692 PMCid:2600445

2. Smith SM, Le Beau MM, Huo D, et al. Clinical-cytogenetic associations in 306 patients with therapy-related myelodysplasia and myeloid leukemia: the University of Chicago series. Blood 2003; 102(1): 43-52. doi:10.1182/blood-2002-11-3343 PMid:12623843

3. Kayser S, Döhner K, Krauter J, et al. The impact of therapyrelated acute myeloid leukemia (AML) on outcome of 2853 adult patients with newly diagnosed AML. Blood 2011; 117(7): 2137 2145. doi:10.1182/blood-2010-08-301713 PMid:21127174

4. Andersen MK, Johansson B, Larsen SO, Pedersen-Bjergaard J. Chromosomal abnormalities in secondary MDS and AML. Relationship to drugs and radiation with specific emphasis on the balanced rearrangements. Haematologica 1998; 83: 483-488. PMid:9676019

5. Larson RA, Le Beau MM, Ratain MJ, Rowley JD. Balanced translocations involving chromosome bands $11 \mathrm{q} 23$ and $21 \mathrm{q} 22$ in therapy-related leukemia. Blood 1992; 79: 1892-1893. PMid: 1558980

6. Schoch C, Kern W, Schnittger, et al. Karyotype is an independent prognostic parameter in therapy-related acute myeloid leukemia (t-AML): an analysis of 93 patients with tAML in comparison to 1091 patients with de novo AML. Leukemia 2004; 18(1):120-125. doi:10.1038/sj.leu.2403187 PMid:14586477

7. Kern W, Haferlach T, Schnittger S, et al. Prognosis in therapy- related acute myeloid leukemia and impact of karyotype. J Clin Oncol 2004 June 15; 22(12): 2510-2511. doi:10.1200/JCO.2004.99.301 PMid:15197216

8. Armand P, Kim HT, DeAngelo DJ, et al. Impact of cytogenetics on outcome of de novo and therapy-related AML and MDS after allogeneic transplantation. Biol Blood Marrow Transplant 2007 June; 13(6): 655-664. doi:10.1016/j.bbmt.2007.01.079 PMid:17531775 PMCid:2743535

9. Detourmignies L, Castaigne S, Stoppa AM, et al. Therapyrelated acute promyelocytic leukemia: a report on 16 cases. J Clin Oncol 1992; 10: 1430-1435. PMid:1517786

10. Rowley JD and Olney HJ. International workshop on the relationship of prior therapy to balanced chromosome aberrations in therapy-related myelodysplastic syndromes and acute leukemia: overview report. Genes Chromos Cancer 2002; 33: 331-345.

11. Andersen MK, Larson RA, Mauritzson N, et al. Balanced chromosome abnormalities $\operatorname{inv}(16)$ and $\mathrm{t}(15 ; 17)$ in therapyrelated myelodysplastic syndromes and acute leukemia: report from an international workshop. Genes Chromos Cancer 2002; 33: 395-400. doi:10.1002/gcc.10043 PMid:11921273

12. Beaumont M, Sanz M, Carli PM, et al. Therapy-related acute promyelocytic leukemia. J Clin Oncol 2003; 21: 2123-2137. doi:10.1200/JCO.2003.09.072 PMid:12775738

13. Jantunen E, Heinonen K, Mahlamaki E, et al. Secondary acute promyelocytic leukemia: an increasingly common entity. Leuk Lymphoma 2007; $\quad 48: \quad 190-191$. doi:10.1080/10428190600961736

14. Dayyani F, Kantarjian H, O'Brien S, et al. Outcome of therapy- 
related acute promyelocytic leukemia with or without arsenic trioxide as a component of frontline therapy. Cancer 2011; 117: 110-115. doi:10.1002/cner.25585 PMid:20803607

15. Malhotra P, Varma N, Arora N, et al. Treatment of therapy related acute promyelocytic leukemia with the combination of all-trans retinoic acid and arsenic trioxide without chemotherapy: a series of three patients. Leuk Lymphoma 2010; 51: 933-936. doi:10.3109/10428191003697484

16. Sanz MA, Grimwade D, Tallman MS, et al. Management of acute promyelocytic leukemia: recommendations from an expert panel on behalf of the European LeukemiaNet. Blood 2009; 113: 1875-91. doi:10.1182/blood-2008-04-150250 PMid:18812465

17. Arber DA, Slovak ML, Popplewell L, et al. Therapy-related acute myeloid leukemia/myelodysplasia with balanced $21 \mathrm{q} 22$ translocations. Am J Clin Pathol 2002; 117: 306-13. doi:10.1309/C3G2-CXA0-HE9J-TKDR PMid:11863228

18. Slovak MK, Bedell V, Popplewell L, et al. 21q22 balanced chromosome aberrations in therapy-related hematopoietic disorders: report from an international workshop. Genes Chromos Cancer 2002; 33: 379-394. doi:10.1002/gcc.10042 PMid:11921272

19. Paschka P, Marcucci G, Ruppert AS, et al. Adverse prognostic significance of KIT mutations in adult acute myeloid leukemia with inv(16) and $t(8 ; 21)$ : a Cancer and Leukemia Group B Study. J Clin Oncol 2006 Aug 20; 24(24): 3904-3911. doi:10.1200/JCO.2006.06.9500 PMid:16921041 